\title{
Spectral characteristics of steady-state Lévy flights in confinement potential profiles
}

\author{
A A Kharcheva ${ }^{1}$, A A Dubkov ${ }^{1}$, B Dybiec ${ }^{2}$, B Spagnolo ${ }^{3,4}$ \\ and $D$ Valenti ${ }^{4}$ \\ ${ }^{1}$ Radiophysical Department, Lobachevsky State University, Gagarin ave. 23, \\ 603950 Nizhni Novgorod, Russia \\ 2 Marian Smoluchowski Institute of Physics, Jagiellonian University, St. \\ Łojasiewicza 11, 30-348 Kraków, Poland \\ ${ }^{3}$ Instituto Nazionale di Fisica Nucleare, Sezione di Catania, Italy \\ ${ }^{4}$ Dipartimento di Fisica e Chimica, Group of Interdisciplinary Theoretical \\ Physics, Università di Palermo and CNISM-Unità di Palermo, Viale delle \\ Scienze, Edificio 18, I-90128 Palermo, Italy \\ E-mail: kharcheva@rf.unn.ru,dubkov@rf.unn.ru, bartek@th.if.uj.edu.pl, \\ bernardo.spagnolo@unipa.it and davide.valenti@unipa.it
}

Received 22 October 2015, revised 24 December 2015

Accepted for publication 11 January 2016

Published 20 May 2016

Online at stacks.iop.org/JSTAT/2016/054039

doi:10.1088/1742-5468/2016/05/054039

\begin{abstract}
The steady-state correlation characteristics of superdiffusion in the form of Lévy flights in one-dimensional confinement potential profiles are investigated both theoretically and numerically. Specifically, for Cauchy stable noise we calculate the steady-state probability density function for an infinitely deep rectangular potential well and for a symmetric steep potential well of the type $U(x) \propto x^{2 m}$. For these potential profiles and arbitrary Lévy index $\alpha$, we obtain the asymptotic expression of the spectral power density.
\end{abstract}

Keywords: rigorous results in statistical mechanics, stochastic particle dynamics, stochastic processes (theory) 


\section{Contents}

1. Introduction 2

2. Mathematical apparatus 3

3. The spectral and correlation characteristics of Brownian diffusion 3

4. The probability and spectral characteristics of Lévy flights 5

5. Conclusions 9

$\begin{array}{ll}\text { Acknowledgments } & 10\end{array}$

$\begin{array}{ll}\text { Appendix } & 11\end{array}$

$\begin{array}{ll}\text { References } & 13\end{array}$

\section{Introduction}

The spectral power densities of fluctuations provide an important tool to characterize physical systems, because they can be measured directly in experiments. Investigations of spectra are useful to observe and analyze the interplay between fluctuations, relaxation and nonlinearity which are inherent to real physical systems. This interplay ranks among the most challenging problems of modern nonlinear physics.

The spectral and correlation characteristics of both overdamped and underdamped Brownian diffusion in fixed and fluctuating one-dimensional potentials have been extensively studied both experimentally and theoretically [1-9].

Anomalous diffusion in the form of Lévy flights is of permanent interest due to wide applications in different areas of science [10-30]. To explore this phenomenon, unlike the standard Brownian motion, one needs to apply the Markovian theory of the fractional Fokker-Planck equation and to state non-trivial boundary conditions. As a result, even the steady-state probability density function of the particle coordinate can be found only for some simple potential profiles [13, 15, 31].

The analytical investigation of correlational and spectral properties of steady-state Lévy flights in confinement potentials remains an open problem. Here we can mention only the exact result, recently obtained in [32], for the correlation time of Lévy flights in a symmetric quartic potential.

The paper is organized as follows. In the next section we start with the mathematical apparatus needed for the analysis of spectral and correlation characteristics of Lévy flights in confinement potentials. In section 3 we briefly review the spectral and correlation characteristics of Brownian diffusion. The probability and spectral characteristics of Lévy flights in confinement potentials are analyzed in section 4 . Finally in section 5 we draw conclusions. 


\section{Mathematical apparatus}

We start from the following general operator formula for the correlation function $K[\tau]$ of a stationary Markovian process $x(t)$ [33]

$$
K[\tau]=\left\langle x \mathrm{e}^{\hat{L}^{+}(x) \tau} x\right\rangle, \quad \tau \geqslant 0
$$

where $\hat{L}^{+}(x)$ is the adjoint kinetic operator of the general Kolmogorov equation for the probability density function.

According to the Wiener-Khinchin theorem, the spectral power density can be found as

$$
S(\omega)=\int_{-\infty}^{\infty} K[\tau] \cos \omega \tau \mathrm{d} \tau=2 \operatorname{Re}\{\tilde{K}[\mathrm{i} \omega]\}
$$

where $\tilde{K}[p]$ is the Laplace transform of $K[\tau]$. According to equation (1) $\tilde{K}[p]$ reads

$$
\tilde{K}[p]=\left\langle x \frac{1}{p-\hat{L}^{+}(x)} x\right\rangle .
$$

As seen from equation (3), we have to solve the following integro-differential equation for the auxiliary function $\varphi_{p}(x)$

$$
\hat{L}^{+}(x) \varphi_{p}(x)-p \varphi_{p}(x)=-x
$$

and then calculate the average

$$
\tilde{K}[p]=\left\langle x \varphi_{p}(x)\right\rangle
$$

over the stationary probability density function.

In particular, using one of the definitions of correlation time (see [34]) we have

$$
\tau_{k}=\frac{1}{\sigma^{2}} \int_{0}^{\infty}\left(K[\tau]-\langle x\rangle^{2}\right) \mathrm{d} \tau=\frac{S\left(0^{+}\right)}{2 \sigma^{2}},
$$

where $\sigma^{2}$ is the variance of the random process $x(t)$.

Based on this mathematical tool, we analyze the steady-state probability and correlation characteristics of Brownian motion, and the anomalous diffusion in the form of Lévy flights in an infinitely deep rectangular potential well.

\section{The spectral and correlation characteristics of Brownian diffusion}

In this section, we briefly review some results concerning the overdamped Brownian motion of a particle in the potential $U(x)$ under the action of white Gaussian noise $\xi(t)$ with zero mean $\langle\xi(t)\rangle=0$ and intensity $2 D$. This motion can be described by the following Langevin equation 


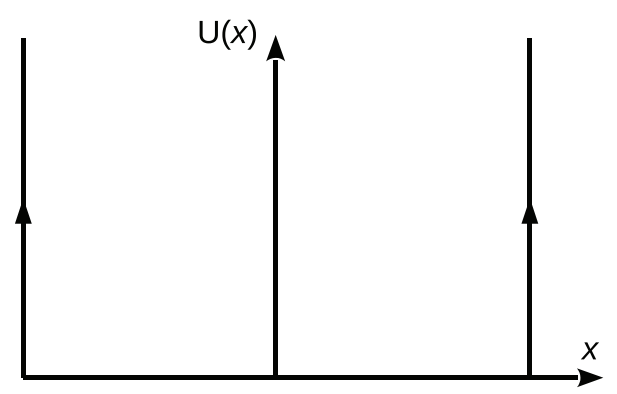

Figure 1. Infinitely deep rectangular potential well.

$$
\frac{\mathrm{d} x}{\mathrm{~d} t}=-\frac{\mathrm{d} U(x)}{\mathrm{d} x}+\xi(t),
$$

where $x(t)$ is the particle coordinate.

The corresponding Fokker-Planck (or Smoluchowski) equation for the probability density function reads

$$
\frac{\partial P}{\partial t}=\frac{\partial}{\partial x}\left(\frac{\mathrm{d} U}{\mathrm{~d} x} P\right)+D \frac{\partial^{2} P}{\partial x^{2}} .
$$

From equation (8) we find the adjoint kinetic operator

$$
\hat{L}^{+}(x)=D \frac{\partial^{2}}{\partial x^{2}}-U^{\prime}(x) \frac{\partial}{\partial x}
$$

and the steady-state probability distribution of particle position

$$
P_{\mathrm{st}}(x)=C_{0} \mathrm{e}^{-U(x) / D},
$$

which is of the Boltzmann-Gibbs type, with the normalization constant $C_{0}$.

In particular, for an infinitely deep rectangular potential well (see figure 1)

$$
U(x)=\left\{\begin{array}{cc}
0, & |x| \leqslant L \\
\infty, & |x|>L
\end{array}\right.
$$

from equation (10) we obtain a uniform probability distribution inside a potential well

$$
P_{\mathrm{st}}(x)=\frac{1}{2 L}, \quad|x|<L
$$

The steady-state spectral power density of the particle position in such a case was found in [8] from equations (2) and (3) as

$$
S(\omega)=\frac{2 D}{\omega^{2}}\left(1-\frac{1}{L} \sqrt{\frac{D}{2 \omega}} \frac{\sinh L \sqrt{2 \omega / D}+\sin L \sqrt{2 \omega / D}}{\cosh L \sqrt{2 \omega / D}+\cos L \sqrt{2 \omega / D}}\right) .
$$


From equation (13) we get

$$
S(\omega) \cong \frac{2 D}{\omega^{2}}, \quad \omega \rightarrow \infty
$$

Further, we show that the result (14) holds for any potential $U(x)$. Indeed, from equations (1), (9) and (10) we find

$$
K^{\prime}\left[0^{+}\right]=\left\langle x \hat{L}^{+}(x) x\right\rangle=-\left\langle x U^{\prime}(x)\right\rangle=-D .
$$

Thus, the first derivative of the stationary correlation function at $\tau=0^{+}$does not depend on the form of potential. According to the Tauberian theorems we have

$$
\begin{aligned}
& K[0]=\sigma^{2}=\lim _{p \rightarrow \infty} p \tilde{K}[p], \\
& K^{\prime}\left[0^{+}\right]=-D=\lim _{p \rightarrow \infty} p\left(p \tilde{K}[p]-\sigma^{2}\right) .
\end{aligned}
$$

and, as a result

$$
\tilde{K}[p] \simeq \frac{\sigma^{2}}{p}-\frac{D}{p^{2}}, \quad p \rightarrow \infty .
$$

Substitution of equation (16) in equation (2) gives the universal asymptotic behavior (14) of the spectral power density. Finally, from equations (6), (12) and (13) we find the correlation time as

$$
\tau_{c}=\frac{2 L^{2}}{5 D}
$$

\section{The probability and spectral characteristics of Lévy flights}

Here, we consider anomalous diffusion in the form of Lévy flights in the potential $U(x)$, which is governed by the following Langevin equation for the particle coordinate $x(t)$

$$
\frac{\mathrm{d} x}{\mathrm{~d} t}=-\frac{\mathrm{d} U(x)}{\mathrm{d} x}+\xi_{\alpha}(t)
$$

where $\xi_{\alpha}(t)$ is a symmetric $\alpha$-stable Lévy noise and $\alpha$ is the Lévy index $(0<\alpha<2)$. The corresponding Fokker-Planck equation with spatial fractional derivative for the probability density function takes the form [12]

$$
\frac{\partial P}{\partial t}=\frac{\partial}{\partial x}\left(\frac{\mathrm{d} U}{\mathrm{~d} x} P\right)+D_{\alpha} \frac{\partial^{\alpha} P}{\partial|x|^{\alpha}},
$$

where $D_{\alpha}$ is the intensity parameter of the noise $\xi_{\alpha}(t)$.

To calculate the steady-state characteristics of Lévy flights in an infinitely deep rectangular potential well, we examine a symmetric steep potential well of the type $U(x) \propto x^{2 m}$. Specifically, we consider the symmetric steep confining potential 


$$
U(x)=\frac{\gamma}{2 m}\left(\frac{x}{L}\right)^{2 m}
$$

For this potential profile, as shown in [15], the stationary probability distribution of the particle displacement for anomalous diffusion in the form of Lévy flights with Lévy index $\alpha=1$ has the following expressions for odd $m=2 n+1$

$$
P_{\mathrm{st}}(x)=\frac{\beta^{4 n+1}}{\pi\left(x^{2}+\beta^{2}\right)} \prod_{l=0}^{n-1} \frac{1}{x^{4}-2 \beta^{2} x^{2} \cos [\pi(4 l+1) /(4 n+1)]+\beta^{4}}
$$

and for even $m=2 n$

$$
P_{\mathrm{st}}(x)=\frac{\beta^{4 n-1}}{\pi} \prod_{l=0}^{n-1} \frac{1}{x^{4}-2 \beta^{2} x^{2} \cos [\pi(4 l+1) /(4 n-1)]+\beta^{4}},
$$

where $\beta=L \sqrt[2 m-1]{D_{1} L / \gamma}$, and $D_{1}$ is the intensity parameter of the noise with stable Cauchy distribution.

In the limit of very large exponent $m$, the potential (20) transforms to an infinitely deep rectangular potential well (11). To make this limit in equations (21) and (22) we need to rearrange them into a more convenient form (see appendix)

$$
P_{\mathrm{st}}(x)=\left\{\begin{array}{cl}
\frac{1}{\pi \beta} \exp \left\{\sum_{k=1}^{\infty} \frac{1}{2 k \cos \frac{\pi k}{2 m-1}}\left(\frac{x}{\beta}\right)^{2 k}\right\}, & |x| \leqslant \beta, \\
\frac{1}{\pi \beta}\left(\frac{\beta}{x}\right)^{2 m} \exp \left\{\sum_{k=1}^{\infty} \frac{1}{2 k \cos \frac{\pi k}{2 m-1}}\left(\frac{\beta}{x}\right)^{2 k}\right\}, & |x|>\beta .
\end{array}\right.
$$

In the limit $m \rightarrow \infty$, the steady-state probability distribution (23) transforms to the arcsine distribution

$$
P_{\mathrm{st}}(x)=\left\{\begin{array}{cc}
\frac{1}{\pi} \frac{1}{\sqrt{L^{2}-x^{2}}}, & |x| \leqslant L, \\
0, & |x|>L .
\end{array}\right.
$$

The validity of this transformation can be confirmed by comparing equation (24) with the exact results given in [35] for arbitrary Lévy index $\alpha$

$$
P_{\mathrm{st}}(x)=\frac{(2 L)^{1-\alpha} \Gamma(\alpha)}{\Gamma^{2}(\alpha / 2)\left(L^{2}-x^{2}\right)^{1-\alpha / 2}},
$$

where $\Gamma(x)$ is the gamma function. The formula (25) was derived by using the special conditions for impermeable boundaries at $x= \pm L$.

The stationary probability densities of equation (25), for different values of the Lévy index $\alpha$, are shown in figure 2 (solid lines). Points in the figure have been obtained by numerically integrating the stochastic differential equation (18), applying the EulerMaruyama method within the Ito scheme [36, 37]. The same procedure has been followed to get all numerical results presented in this paper. More precisely, a large 


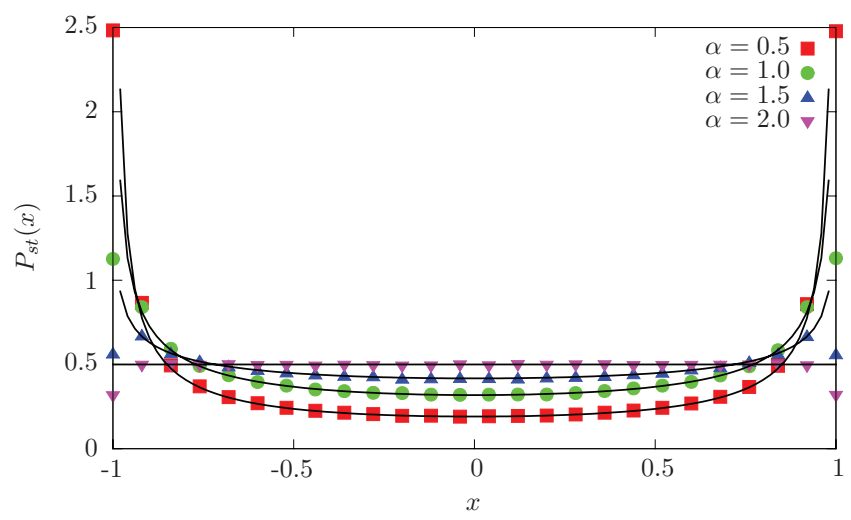

Figure 2. Stationary probability densities $P_{\mathrm{st}}(x)$ for different values of the Lévy index $\alpha$. The values of the parameters are: $\gamma=1, D_{\alpha}=1$ and $L=1$. The case $\alpha=2$ corresponds to usual Brownian motion. Solid lines represent exact solutions given by equation (25), while points correspond to numerical estimations of stationary distributions based on the Langevin equation (18). The numerical simulations are obtained using the potential profile of equation (20) with $m=800$.

number of very long realizations of the stochastic process $x(t)$ is generated. From these realizations the time dependent densities $P(x, t)$ are estimated. Finally, the stationary density $P_{\mathrm{st}}(x)=\lim _{t \rightarrow \infty} P(x, t)$ is approximated. The curve with $\alpha=2$ corresponds to the usual Brownian motion. In such a case the stationary density is of the BoltzmannGibbs type for the infinitely deep rectangular potential well (see equation (12)). The infinitely deep rectangular potential well can be achieved from equation (20) in the limit of $m \rightarrow \infty$. Practically, such a convergence is quite fast and with $m=800$ we observe a very good agreement between numerical simulations and exact formula (25), except the points close to the boundaries (see figure 2). Alternatively, the infinitely deep rectangular potential well can be approximated by imposing impenetrable boundary conditions on trajectories $x(t)$, see [35].

In figure 3 we show the stationary probability density functions for $\alpha=1$, obtained with increasing exponent $m$ (see equation (20)). Exact results, which are given by equations (21) and (22), are depicted by solid lines. Points correspond to results of Monte Carlo simulations of the Langevin equation (18). In the limit of $m \rightarrow \infty$, the stationary probability density function tends to the arcsine distribution of equation (24). This is achieved for $m=50$. Figure 3 demonstrates how, starting from a steep potential, we can get the stationary probability distributions in the infinitely deep rectangular potential well, without considering the problem of the boundary conditions. The steady-state spectral characteristics of Lévy flights, with potential profile of equation (20), cannot be obtained analytically for arbitrary Lévy index $\alpha$.

However, an asymptotic expression of the spectral power density of steady-state Lévy flights in the potential (20) can be found. From equations (1), (19), (20) and (23) we get the first derivative of the correlation function at zero point

$$
K^{\prime}\left[0^{+}\right]=\left\langle x \hat{L}^{+}(x) x\right\rangle=-\left\langle x U^{\prime}(x)\right\rangle=-\gamma\left\langle\left(\frac{x}{L}\right)^{2 m}\right\rangle=-\infty
$$

which differs from the result (15) for ordinary Brownian motion $(\alpha=2)$. 
Spectral characteristics of Lévy flights in confinement potentials

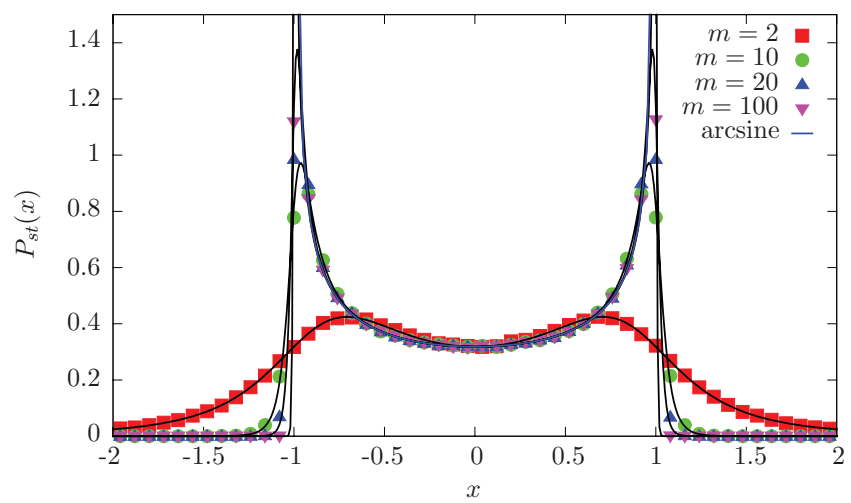

Figure 3. Stationary probability distributions for $\alpha=1$ and increasing exponent $m$ (see equations (20)-(22)). Solid lines represent exact results, while points correspond to numerical estimations of stationary densities based on the Langevin equation (18). The values of the other system parameters are the same as those of figure 2.

Figure 4 presents sample autocorrelation functions $K[\tau]$ for $\alpha=1.9$ (top panel) and $\alpha=1.5$ (bottom panel) plotted in two different scales, namely as $K[\tau]$ versus $\tau$ (left side panels) and $\ln |\ln K[\tau]|$ versus $\ln \tau$ (right side panels). Various curves correspond to different values of the exponent $m$ (see equation (20)).

In accordance with equation (26), we can assume a non-analytical dependence of the correlation function on $\tau$ near the point $\tau=0$ in the following ansatz form

$$
K[\tau] \simeq \sigma^{2}\left[1-\left(\frac{\tau}{\tau_{0}}\right)^{\nu}\right] \simeq \sigma^{2} \exp \left\{-\left(\frac{\tau}{\tau_{0}}\right)^{\nu}\right\}, \quad 0<\nu<1, \quad \tau \ll \tau_{0} .
$$

Applying a Laplace transform to equation (27) we arrive at

$$
\tilde{K}[p] \simeq \sigma^{2}\left[\frac{1}{p}-\frac{\Gamma(1+\nu)}{\tau_{0}^{\nu} p^{1+\nu}}\right], \quad p \rightarrow \infty .
$$

Substitution of equation (28) into equation (2) gives the asymptotic expression of the spectral power density

$$
S(\omega) \simeq \frac{2 \sigma^{2} \Gamma(1+\nu) \sin (\pi \nu / 2)}{\tau_{0}^{\nu} \omega^{1+\nu}}, \quad \omega \rightarrow \infty .
$$

The exponent $\nu$ is a function of the potential exponent $m$, the noise intensity parameter $D_{\alpha}$, the Lévy index $\alpha$, the steepness of potential $\gamma$ and the size of the potential well $L$.

In the left side panels of figure 4 we also show the best fitting of the normalized correlation function $K[\tau]$, obtained by numerical simulation of the Langevin equation (18) with the potential profile of equation (20) calculated at different values of exponent $m$. From the fitting procedure we obtain the values of the parameters $\sigma^{2}, \tau_{0}$ and $\nu$ of the stretched exponential function of equation (27) shown in table 1.

In figure 5 we show the spectral power density obtained by numerical simulation of the Langevin equation (18) with the potential profile of equation (20) for $m=100$, and four different values of the Lévy index $\alpha$, namely $\alpha=1.3,1.5,1.9,2.0$. In all simulations 
Spectral characteristics of Lévy flights in confinement potentials
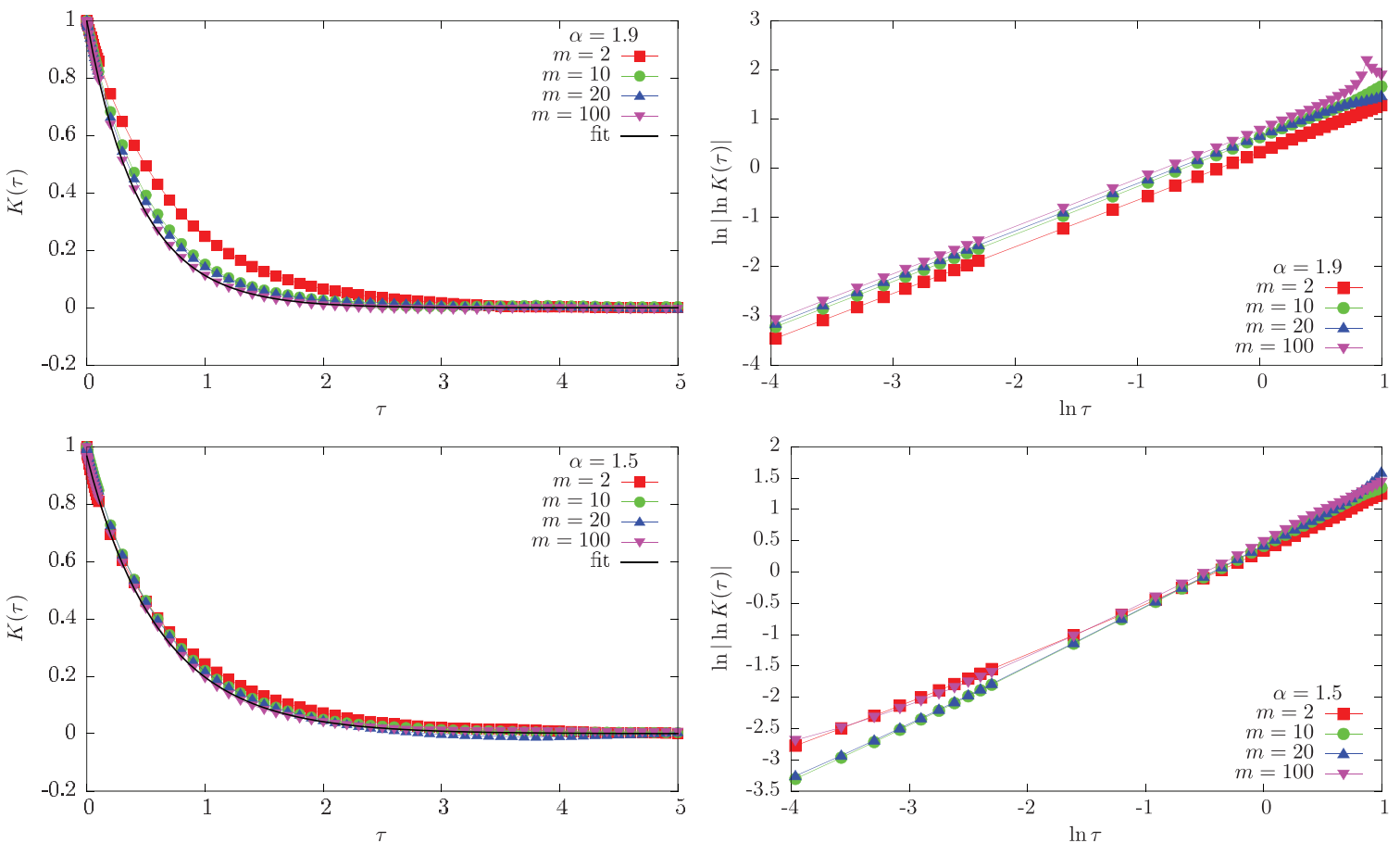

Figure 4. Correlation function for $\alpha=1.9$ (top panels), $\alpha=1.5$ (bottom panels) plotted in two different scales, namely as $K[\tau]$ versus $\tau$ (left side panels) and $\ln |\ln K[\tau]|$ versus $\ln \tau$ (right side panels). Various curves correspond to different values of the exponent $m$ (see equation (20)). The values of the other system parameters are the same as those of figure 2 .

Table 1. Values of stretched exponential parameters fitted to the normalized correlation function.

\begin{tabular}{llll}
\hline$\alpha$ & $\sigma^{2}$ & $\tau_{0}$ & $\nu$ \\
\hline 1.3 & $0.950 \pm 0.001$ & $0.702 \pm 0.003$ & $0.987 \pm 0.006$ \\
1.5 & $0.967 \pm 0.001$ & $0.622 \pm 0.002$ & $0.980 \pm 0.004$ \\
1.9 & $0.999 \pm 0.001$ & $0.452 \pm 0.002$ & $0.984 \pm 0.005$ \\
\hline
\end{tabular}

we fixed a noise intensity parameter to $D_{\alpha}=1$. The integration step used to obtain the curves of figure 5 was $\Delta t=10^{-6}$, and the obtained spectral power densities were averaged over 300 realizations. Moreover, for $\alpha=1.3,1.5,1.9$ we also show the spectral power densities obtained by a Fourier transform of the stretched exponential ansatz of equation (27). The agreement between these curves and those calculated by direct simulation of the Langevin equation (18) is very good.

\section{Conclusions}

We have investigated the spectral characteristics of steady-state Lévy flights in confinement potential profiles. The main results of the paper are: (i) the analytical expression of the steady-state probability density function for an infinitely deep 


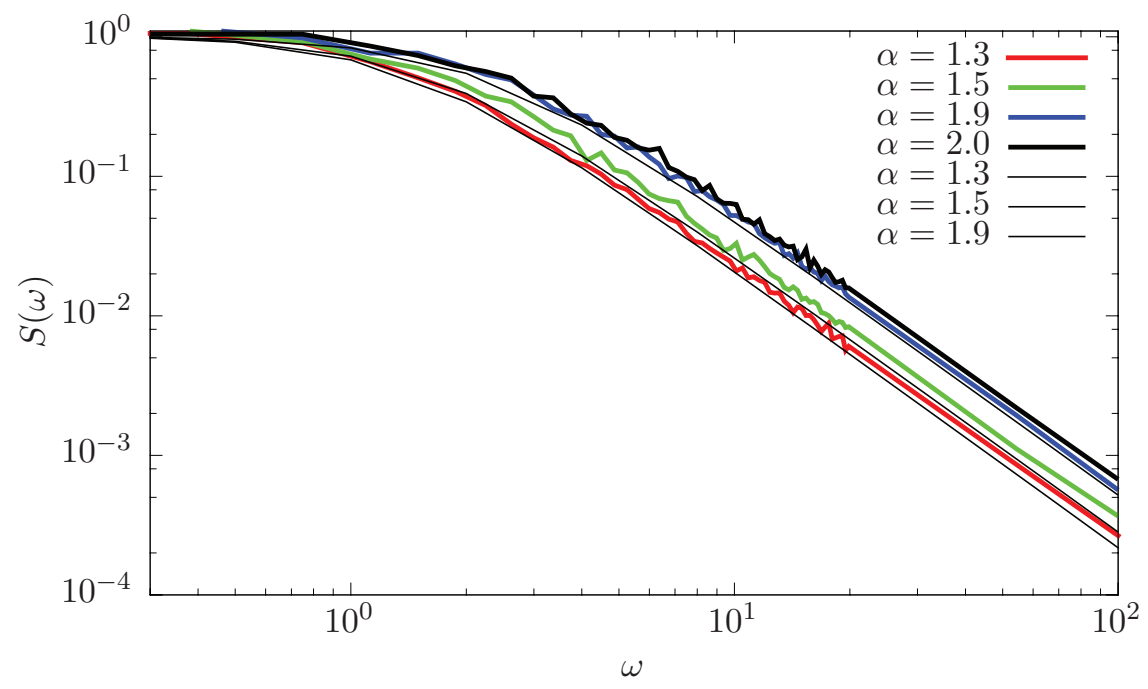

Figure 5. Color lines represent spectral power densities estimated from time series of $x(t)$, obtained solving equation (18) for four different values of the Lévy index $\alpha$. Thin black lines (solid, medium-dashed, and short-dashed) refer to spectral power densities for $\alpha=1.3,1.5$ and 1.9 estimated starting from the fitted stretched exponential given in equation (27) and calculating its Fourier transform. Trajectories have been constructed by the Langevin dynamics with the integration step $\Delta t=10^{-6}$. Every trajectory $x(t)$ consists of $2^{24}$ elements. Spectral power densities has been averaged over 300 realizations. The $\alpha$-stable noise intensity parameter is $D_{\alpha}=1$. The exponent in the steep potential (20) is $m=100$.

rectangular potential well and for a symmetric steep potential well of the type $U(x) \propto x^{2 m}$, in the case of Lévy noise with Cauchy-Lorentz distribution $(\alpha=1)$; (ii) the asymptotic expression of the spectral power density for superdiffusion in symmetric steep potential profiles, for arbitrary Lévy noise index $\alpha$. The theoretical results obtained for anomalous diffusion, that is superdiffusion in the form of Lévy flights, in confinement potential profiles are compared with those found for normal Brownian diffusion. Numerical simulations are in a very good agreement with the analytical results. Our study, describing confined Lévy flights, that is Lévy flights with finite variance, could be useful to describe the distribution of flying objects in spatially limited structures, such as confined plasmas and turbulent flows [35].

\section{Acknowledgments}

This project has been supported in part by a grant from the National Science Center (2014/13/B/ST2/02014). Computer simulations have been performed at the Academic Computer Center Cyfronet, Akademia Górniczo-Hutnicza (Kraków, Poland).

This work was supported by the Ministry of Education and Science of the Russian Federation through Agreement No. 14.578.21.0033 (RFMEFI57814X0033), and Ministry of Education, University and Research of Italian Government. 


\section{Appendix}

First of all, we rearrange equation (21) for the case of odd $m=2 n+1$. For convenience, we introduce new symbol $A_{l}=(4 l+1) /(4 n+1)$ and start from the factorization of the denominator in equation (21)

$$
x^{4}-2 \beta^{2} x^{2} \cos \pi A_{l}+\beta^{4}=\left(\beta^{2}-x^{2} \mathrm{e}^{-\mathrm{i} \pi A_{l}}\right)\left(\beta^{2}-x^{2} \mathrm{e}^{\mathrm{i} \pi A_{l}}\right) .
$$

Then we transform in equation (21) the product to the sum in the following way

$$
\begin{aligned}
P_{\text {st }}(x) & =\frac{\beta^{4 n+1}}{\pi\left(x^{2}+\beta^{2}\right)} \prod_{l=0}^{n-1} \frac{1}{\left(\beta^{2}-x^{2} \mathrm{e}^{-\mathrm{i} \pi A_{l}}\right)\left(\beta^{2}-x^{2} \mathrm{e}^{\mathrm{i} \pi A_{l}}\right)} \\
& =\frac{\beta^{4 n+1}}{\pi\left(x^{2}+\beta^{2}\right)} \exp \left\{-\sum_{l=0}^{n-1}\left[\ln \left(\beta^{2}-x^{2} \mathrm{e}^{-\mathrm{i} \pi A_{l}}\right)+\ln \left(\beta^{2}-x^{2} \mathrm{e}^{\mathrm{i} \pi A_{l}}\right)\right]\right\} .
\end{aligned}
$$

In accordance with the Taylor expansion of the logarithm, for $|x|<\beta$ we have

$$
\begin{aligned}
\ln \left(\beta^{2}-x^{2} \mathrm{e}^{-\mathrm{i} \pi A_{l}}\right) & =\ln \beta^{2}+\ln \left(1-\frac{x^{2} \mathrm{e}^{-\mathrm{i} \pi A_{l}}}{\beta^{2}}\right) \\
& =\ln \beta^{2}-\sum_{k=1}^{\infty} \frac{1}{k}\left(\frac{x}{\beta}\right)^{2 k} \mathrm{e}^{-\mathrm{i} \pi k A_{l}} .
\end{aligned}
$$

By analogy

$$
\ln \left(\beta^{2}-x^{2} \mathrm{e}^{\mathrm{i} \pi A_{l}}\right)=\ln \beta^{2}-\sum_{k=1}^{\infty} \frac{1}{k}\left(\frac{x}{\beta}\right)^{2 k} \mathrm{e}^{\mathrm{i} \pi k A_{l}} .
$$

Substituting these relations in equation (A.2) we find

$$
\begin{aligned}
P_{\mathrm{st}}(x) & =\frac{\beta^{4 n+1}}{\pi\left(x^{2}+\beta^{2}\right)} \exp \left\{\sum_{l=0}^{n-1}\left[\ln \frac{1}{\beta^{4}}+\sum_{k=1}^{\infty} \frac{1}{k}\left(\frac{x}{\beta}\right)^{2 k}\left(\mathrm{e}^{\mathrm{i} \pi k A_{l}}+\mathrm{e}^{-\mathrm{i} \pi k A_{l}}\right)\right]\right\} \\
& =\frac{\beta}{\pi\left(x^{2}+\beta^{2}\right)} \exp \left\{\sum_{k=1}^{\infty} \frac{1}{k}\left(\frac{x}{\beta}\right)^{2 k} \sum_{l=0}^{n-1}\left(\mathrm{e}^{\mathrm{i} \pi k \frac{4 l+1}{4 n+1}}+\mathrm{e}^{-\mathrm{i} \pi k \frac{4 l+1}{4 n+1}}\right)\right\} .
\end{aligned}
$$

To calculate the internal sum on index $l$, we use the formula for the sum of the geometric series

$$
\begin{aligned}
\sum_{l=0}^{n-1}\left(\mathrm{e}^{\mathrm{i} \pi k \frac{4 l+1}{4 n+1}}+\mathrm{e}^{-\mathrm{i} \pi k \frac{4 l+1}{4 n+1}}\right) & =2 \operatorname{Re}\left\{\sum_{l=0}^{n-1}\left(\mathrm{e}^{\frac{\mathrm{i} \pi k}{4 n+1}}\right)^{4 l+1}\right\} \\
& =2 \operatorname{Re}\left\{\frac{\mathrm{e}^{\frac{\mathrm{i} \pi k}{4 n+1}-\mathrm{e}^{\mathrm{i} \pi k}}}{1-\frac{4 \pi k k}{4 n+1}}\right\}=\operatorname{Re}\left\{\mathrm{i} \frac{\mathrm{e}^{-\frac{\mathrm{i} \pi k}{4 n+1}}+(-1)^{k+1} \mathrm{e}^{-\frac{2 i \pi k}{4 n+1}}}{\sin \frac{2 \pi k}{4 n+1}}\right\} \\
& =\frac{\sin \frac{\pi k}{4 n+1}}{\sin \frac{2 \pi k}{4 n+1}}+(-1)^{k+1}=\frac{1}{2 \cos \frac{\pi k}{2 m-1}}+(-1)^{k+1}
\end{aligned}
$$


Substituting (A.4) in (A.3), we arrive at

$$
\begin{aligned}
P_{\mathrm{st}}(x) & =\frac{\beta}{\pi\left(x^{2}+\beta^{2}\right)} \exp \left\{\sum_{k=1}^{\infty}\left[\frac{1}{2 k \cos \frac{\pi k}{2 m-1}}\left(\frac{x}{\beta}\right)^{2 k}+\frac{(-1)^{k+1}}{k}\left(\frac{x}{\beta}\right)^{2 k}\right]\right\} \\
& =\frac{\beta}{\pi\left(x^{2}+\beta^{2}\right)} \exp \left\{\ln \left[1+\left(\frac{x}{\beta}\right)^{2}\right]+\sum_{k=1}^{\infty} \frac{1}{2 k \cos \frac{\pi k}{2 m-1}}\left(\frac{x}{\beta}\right)^{2 k}\right\} \\
& =\frac{1}{\pi \beta} \exp \left\{\sum_{k=1}^{\infty} \frac{1}{2 k \cos \frac{\pi k}{2 m-1}}\left(\frac{x}{\beta}\right)^{2 k}\right\} .
\end{aligned}
$$

The formula for even $m=2 n$ can be obtained analogously from equation (22), but we have to replace $A_{l}$ with $B_{l}=(4 l+1) /(4 n-1)$. As a result, we arrive at

$$
\begin{aligned}
P_{\mathrm{st}}(x) & =\frac{\beta^{4 n-1}}{\pi} \exp \left\{\sum_{l=0}^{n-1}\left[\ln \frac{1}{\beta^{4}}+\sum_{k=1}^{\infty} \frac{1}{k}\left(\frac{x}{\beta}\right)^{2 k}\left(\mathrm{e}^{\mathrm{i} \pi k B_{l}}+\mathrm{e}^{-\mathrm{i} \pi k B_{l}}\right)\right]\right\} \\
& =\frac{1}{\pi \beta} \exp \left\{\sum_{k=1}^{\infty} \frac{1}{k}\left(\frac{x}{\beta}\right)^{2 k} \sum_{l=0}^{n-1}\left(\mathrm{e}^{\mathrm{i} \pi k \frac{4 l+1}{4 n-1}}+\mathrm{e}^{-\mathrm{i} \pi k \frac{4 l+1}{4 n-1}}\right)\right\} .
\end{aligned}
$$

Next we calculate the internal sum on index $l$ in equation (A.6) by the same method as in equation (A.4). Namely,

$$
\begin{aligned}
\sum_{l=0}^{n-1}\left(\mathrm{e}^{\mathrm{i} \pi k \frac{4 l+1}{4 n-1}}+\mathrm{e}^{-\mathrm{i} \pi k \frac{4 l+1}{4 n-1}}\right) & =2 \operatorname{Re}\left\{\sum_{l=0}^{n-1}\left(\mathrm{e}^{\frac{\mathrm{i} \pi k}{4 n-1}}\right)^{4 l+1}\right\}=2 \operatorname{Re}\left\{\frac{\mathrm{e}^{\frac{\mathrm{i} \pi k}{4 n-1}}-\mathrm{e}^{\mathrm{i} \pi k} \mathrm{e}^{\frac{2 i \pi k}{4 n-1}}}{1-\mathrm{e}^{\frac{4 i \pi k}{4 n-1}}}\right\} \\
& =\operatorname{Re}\left\{\mathrm{i} \frac{\mathrm{e}^{-\frac{\mathrm{i} \pi k}{4 n-1}}+(-1)^{k+1}}{\sin \frac{2 \pi k}{4 n-1}}\right\}=\frac{1}{2 \cos \frac{\pi k}{2 m-1}} .
\end{aligned}
$$

Substituting equation (A.7) in equation (A.6) we again obtain the result (A.5), i.e.

$$
P_{\mathrm{st}}(x)=\frac{1}{\pi \beta} \exp \left\{\sum_{k=1}^{\infty} \frac{1}{2 k \cos \frac{\pi k}{2 m-1}}\left(\frac{x}{\beta}\right)^{2 k}\right\} .
$$

Using similar calculations, it is easy to obtain the steady-state probability distribution for the case $|x|>\beta$ for odd and even values of the parameter $m$. The final expression for arbitrary $m$ is of the form

$$
P_{\mathrm{st}}(x)=\frac{1}{\pi \beta}\left(\frac{\beta}{x}\right)^{2 m} \exp \left\{\sum_{k=1}^{\infty} \frac{1}{2 k \cos \frac{\pi k}{2 m-1}}\left(\frac{\beta}{x}\right)^{2 k}\right\} .
$$


Finally, from equations (21) and (22) we arrive at

$$
P_{\mathrm{st}}(x)=\left\{\begin{array}{cl}
\frac{1}{\pi \beta} \exp \left\{\sum_{k=1}^{\infty} \frac{1}{2 k \cos \frac{\pi k}{2 m-1}}\left(\frac{x}{\beta}\right)^{2 k}\right\}, & |x| \leqslant \beta, \\
\frac{1}{\pi \beta}\left(\frac{\beta}{x}\right)^{2 m} \exp \left\{\sum_{k=1}^{\infty} \frac{1}{2 k \cos \frac{\pi k}{2 m-1}}\left(\frac{\beta}{x}\right)^{2 k}\right\}, & |x|>\beta .
\end{array}\right.
$$

\section{References}

[1] Caughey T K and Dienes J K 1961 J. Appl. Phys. 322476

[2] Mörsch M, Risken H and Vollmer H D 1979 Z. Phys. B 32245

[3] Dykman M I, Mannella R, McClintock P V E, Moss F and Soskin S M 1988 Phys. Rev. A 371303

[4] Dykman M I, Mannella R, McClintock P V E, Soskin S M and Stocks N G 1990 Phys. Rev. A 427041

[5] Dykman M I, Mannella R, McClintock P V E, Soskin S M and Stocks N G 1991 Phys. Rev. A 431701

[6] Marchesoni F, Menichella-Saetta E, Pochini M and Santucci S 1988 Phys. Rev. A 373058

[7] Ouyang H F, Huang Z Q and Ding E J 1994 Phys. Rev. E 502491

[8] Dubkov A A, Ganin V N and Spagnolo B 2004 Acta Phys. Pol. B 351447

[9] Chichigina O, Valenti D and Spagnolo B 2005 Fluct. Noise Lett. 5 L243

[10] Mantegna R N and Stanley H E 1995 Nature 37646

[11] Ditlevsen P D 1999 Geophys. Res. Lett. 261441

[12] Metzler R and Klafter J 2000 Phys. Rep. 3391

[13] Chechkin A V, Gonchar V Y, Klafter J, Metzler R and Tanatarov L V 2004 J. Stat. Phys. 1151505

[14] Chechkin A V, Gonchar V Y, Klafter J and Metzler R 2006 Fundamentals of Lévy flight processes Advances in Chemical Physics vol 133, ed Y P Kalmykov, W T Coffey and S A Rice (New York: Wiley) chapter 9

[15] Dubkov A A and Spagnolo B 2007 Acta Phys. Pol. B 381745

[16] Reynolds A M 2008 Ecology 892347

[17] Dubkov A A, Spagnolo B and Uchaikin V V 2008 Int. J. Bifurcation Chaos 182649

[18] Uchaikin V V 2003 Phys.-Usp. 46821

[19] Dubkov A A, La Cognata A and Spagnolo B 2009 J. Stat. Mech. P01002

[20] Reynolds A M 2009 J. Phys. A: Math. Theor. 42434006

[21] Dubkov A A and Spagnolo B 2008 Eur. Phys. J. B 65361

[22] Sims D W et al 2009 Nature 4511098

[23] Brockmann D, Hufnagel L and Geisel T 2006 Nature 439462

[24] Augello G, Valenti D and Spagnolo B 2010 Eur. Phys. J. B 78225

[25] Chechkin A V, Gonchar V Y, Gorenflo R, Korabel N and Sokolov I M 2008 Phys. Rev. E 78021111

[26] Dybiec B, Gudowska-Nowak E and Sokolov I M 2007 Phys. Rev. E 76041122

[27] Dybiec B, Sokolov I M and Chechkin A V 2010 J. Stat. Mech P07008

[28] La Cognata A, Valenti D, Dubkov A A and Spagnolo B 2010 Phys. Rev. E 82011121

[29] Palyulin V V, Chechkin A V and Metzler R 2014 Proc. Natl Acad. Sci. USA 1112931

[30] Valenti D, Guarcello C and Spagnolo B 2014 Phys. Rev. B 89214510

[31] Chechkin A, Gonchar V, Klafter J, Metzler R and Tanatarov L 2002 Chem. Phys. 284233

[32] Dubkov A A and Spagnolo B 2013 Eur. Phys. J. Spec. Top. 21631

[33] Medvedev S Y 1977 Radiophys. Quantum Electron. 20863

[34] Dubkov A A, Malakhov A N and Saichev A I 2000 Radiophys. Quantum Electron. 43335

[35] Denisov S I, Horsthemke W and Hänggi P 2008 Phys. Rev. E 77061112

[36] Janicki A and Weron A 1994 Simulation and Chaotic Behavior of $\alpha$-Stable Stochastic Processes (New York: Dekker)

[37] Higham D J 2001 SIAM Rev. 43525 